\title{
A Linear-Time Algorithm to Compute the Conjugate of Convex Piecewise Linear-Quadratic Bivariate Functions
}

\author{
Tasnuva Haque, Yves Lucet
}

January 20, 2018

\begin{abstract}
We propose the first algorithm to compute the conjugate of a bivariate Piecewise LinearQuadratic (PLQ) function in optimal linear worst-case time complexity. The key step is to use a planar graph, called the entity graph, not only to represent the entities (vertex, edge, or face) of the domain of a PLQ function but most importantly to record adjacent entities. We traverse the graph using breadth-first search to compute the conjugate of each entity using graph-matrix calculus, and use the adjacency information to create the output data structure in linear time.

Keywords. Legendre-Fenchel transform; Conjugate; Piecewise linear-quadratic functions; Subdifferential; Convex Function; Computational Convex Analysis (CCA); Computer-Aided Convex Analysis.
\end{abstract}

\section{Introduction}

Convex conjugate functions play a significant role in duality theory. Consider the primal optimization problem

$$
p=\inf _{x \in \mathbb{R}^{d}}[f(x)+g(A x)],
$$

whose Fenchel dual optimization problem is

$$
d=\sup _{y \in \mathbb{R}^{d}}\left[-f^{*}\left(A^{T} y\right)-g^{*}(-y)\right]
$$

where $f^{*}$ denotes the (Fenchel) conjugate of $f$, and $A^{T}$ the transpose of the matrix $A$. Fenchel's duality Theorem states that under some constraint qualification conditions, $p=d$ and the solution of one problem can be computed from the solution of the other. Understanding how to compute the conjugate of a function is one of the key steps to solve a dual optimization problem [Her16].

Computing the conjugate efficiently is also important for regularization. There is a close relationship [Luc06] between the conjugate and the Moreau envelope (also known as Moreau-Yosida approximate or Moreau-Yosida regularization)

$$
M_{\lambda}(s)=\inf _{x \in \mathbb{R}^{d}}\left[f(x)+\frac{\|x-s\|^{2}}{2 \lambda}\right] .
$$

The Moreau envelope regularizes a nonsmooth function while keeping the same local minima [Luc10, PW16]. The set of point at which the infimum is attained is called the proximal mapping and is the basis of many convex and nonconvex optimization techniques like bundle methods [HUL93].

The importance of conjugate functions inspired us to develop an optimal worst-case time algorithm to compute the entire graph of the conjugate to better understand its structure, and gain 
more intuition in the information it carries. Such computation belongs to the field of Computational Convex Analysis (CCA) by contrast with computing the conjugate at a single point, which is an optimization problem.

CCA focuses on creating efficient algorithms to compute transforms commonly encountered in convex analysis: addition, scalar multiplication, Moreau envelope, and (Legendre-Fenchel) conjugate. Those transforms are considered the core convex transforms [GL13, Luc10] and have been used to develop new theories and results in convex optimization [Luc13b]. One application of CCA is Computer-Aided Convex Analysis, which focuses on visualizing transforms applied to lowdimensional functions.

The Computational Convex Analysis (CCA) toolbox has been developed in the past decade to implement the latest algorithms to compute convex transforms efficiently. It is a free and open source Scilab [Con94] toolbox. The CCA toolbox is almost complete for univariate functions but lacking for bivariate functions.

The first algorithm to compute the conjugate of a convex bivariate PLQ function was developed in [GL13] and implemented in Scilab using the Computational Geometry Library CGAL [CGA]. It uses a planar arrangement to store the entities (vertices, edges and faces) of the domain of a PLQ function. The dual arrangement is then computed by looping through the the vertices and computing the conjugate of the associated edges using a decision tree that enumerates all the possibilities. The algorithm uses a binary search tree to detect duplicate points, which results in a $\mathcal{O}(N \log N)$ worst-case time complexity for a function with $N$ pieces. It achieves a linear-time expected case complexity when implemented using a hash table but needs to assume bounded bit length to achieve linear-time worst-case time complexity when implemented using a trie data structure (a try - the name comes from retrieval but is pronounced "try" - is a " $M$-ary tree, whose nodes are $M$-place vectors with components correspoinding to digits or characters" [Knu73, Section 6.3, p. 492]). Our proposed algorithm is simpler and achieves the same performance without such assumption.

For a convex bivariate PLQ function, the full conjugate can be computed using the partial conjugate, which is the conjugate with respect to only one of the variables. Partial conjugates are also PLQ functions but not necessarily convex [Roc70], e.g. the partial conjugate of the $l_{1}$ norm $l_{1}\left(x_{1}, x_{2}\right)=\left|x_{1}\right|+\left|x_{2}\right|$ is $\left(l_{1}\right)_{1}^{*}\left(s_{1}, x_{2}\right)=\iota_{[-1,1]}\left(s_{1}\right)-\left|x_{2}\right|$. In [GJL14], a simpler algorithm than [GL13] is proposed to compute the full conjugate of a bivariate PLQ function by using two partial conjugates; both the full and partial conjugate algorithms achieve the same complexity.

Another algorithm, based on parametric programming, was developed in [Jak13] to compute the conjugate of convex bivariate PLQ functions. It combines a Parametric Quadratic programming (pQP) approach with computational convex analysis. The input and output PLQ functions are stored using a list representation which is internally converted into a face-constraints adjacency representation. While a planar graph was used to store the entities of a PLQ function, the adjacency information of the entities was not stored. That algorithm computes the vertices, which requires a log-linear time complexity in the worst-case. The time required to compute the remaining entities, i.e. rays and segments, is log-quadratic because for every vertex, the algorithm loops through all adjacent edges. However, the worst-case time complexity can be improved [Jak13, Theorem 5.7] by using a half-edge data structure to store the vertices in order. Even with this improvement, the algorithm still needs to detect duplicate entities and only achieves a log-linear worst-case time asymptotic complexity.

A summary of the algorithms for computing the conjugate of a bivariate PLQ function is presented in Table 1, which shows that no algorithm is known so far to compute the conjugate of a bivariate PLQ function in linear worst-case time. We present such a new algorithm in the present paper. To our knowledge, it is the first linear-time algorithm. We implement our algorithm in 
Table 1: The worst-case time complexity of algorithms developed for computing the conjugate of a bivariate PLQ function. All algorithms achieve linear worst-case space complexity.

\begin{tabular}{lcccc}
\hline Algorithm & Source & Time & Data Structure & Restriction \\
\hline Full conjugate & & Log-linear & Red-Black Tree & \\
\cline { 3 - 5 } \begin{tabular}{l} 
(geometric algorithm) \\
\cline { 3 - 5 }
\end{tabular} & {$[$ GL13] } & Linear & Trie & bounded bit length \\
\cline { 3 - 5 } & & Linear & Hash table & Expected time \\
\hline $\begin{array}{l}\text { Full conjugate } \\
\text { (parametric optimization) }\end{array}$ & [Jak13] & Log-linear & List & \\
\hline $\begin{array}{l}\text { Partial conjugate } \\
\text { (geometric algorithm) }\end{array}$ & [GJL14] & Log-linear & Red-Black Tree & \\
\hline
\end{tabular}

Scilab [Con94].

There are two steps to compute the conjugate: a local step that requires computing dual entities one by one, and a global step that input those dual entities into the output data structure while preventing duplicates. Our contribution is twofold. First, for the local step, we propose a simpler approach that uses graph-matrix calculus [Goe08, GL11] instead of a geometric [GL13, GJL14] or a parametric optimization [Jak13] approach. That improvement makes the algorithm simpler but does not improve the worst-case time complexity. Our second contribution improves the global step by using a neighborhood graph to build the output data structure instead of performing a search using a binary search tree (Red-Black tree), a trie, or a hash table [GL13, GJL14, Jak13]. By using additional information about the conjugate, we are able to avoid any search step completely and achieve an optimal linear worst-case time complexity.

This paper is organized as follows. All the basic notations and definitions required to explain our algorithm are included in Section 2. We include a detailed explanation of the data structure we used in Section 3. Our proposed algorithm is explained with an example in Section 4. The complexity of the algorithm is included in Section 5. Section 6 concludes the paper.

\section{Preliminaries and Notations}

First, we fix our definitions and notations. We note ri $C$ (resp. $\operatorname{int} C, \operatorname{co} C$ ) the relative interior (resp. the interior, the convex hull) of a set $C$, and $\operatorname{dom} f$ the effective domain of a function $f: \mathbb{R}^{d} \rightarrow \mathbb{R} \cup\{+\infty\}$ i.e. the set of points where $f$ is finite.

Definition 2.1 (Conjugate function). Consider a proper convex lower semi-continuous (lsc) function $f: \mathbb{R}^{d} \rightarrow \mathbb{R} \cup\{+\infty\}$. The conjugate of $f$, denoted by $f^{*}$, is defined as

$$
f^{*}(s)=\sup _{x}(\langle s, x\rangle-f(x)) .
$$

Definition 2.2 (Polyhedral set). A polyhedral set $C$ in $\mathbb{R}^{d}$ is the intersection of finitely many half-spaces and is denoted $C=\left\{x \in \mathbb{R}^{d}: a_{i}{ }^{T} x \leq b_{i}\right\}$ where $a_{i} \in \mathbb{R}^{d}, b_{i} \in \mathbb{R}, i=1, \ldots, m$.

Definition 2.3 (Proper face). A face of a convex set $C$ is a nonempty subset, $F \subseteq C$ such that if $x_{1}, x_{2} \in C$, and for all $\theta, 0<\theta<1$ we have $\theta x_{1}+(1-\theta) x_{2} \in F$ then $x_{1}, x_{2} \in F$. A face $F$ that is strictly smaller than $C$ is called a proper face. 
Definition 2.4 (Polyhedral decomposition [PS11, Definition 1]). The set $\mathcal{C}=\left\{C_{k}: k \in \mathcal{K}\right\}$, where $\mathcal{K}$ is a finite index set, is called a polyhedral decomposition of $\mathcal{D} \subseteq \mathbb{R}^{d}$ if it satisfies the following conditions

(i) all of its members $C_{k}$ are polyhedral sets,

(ii) $\bigcup_{k \in \mathcal{K}} C_{k}=\mathcal{D}$,

(iii) for all $k \in \mathcal{K}, \operatorname{dim} C_{k}=\operatorname{dim} \mathcal{D}$,

(iv) ri $C_{k_{1}} \cap$ ri $C_{k_{2}}=\emptyset$, where $k_{1}, k_{2} \in \mathcal{K}, k_{1} \neq k_{2}$.

Definition 2.5 (Polyhedral subdivision [PS11, Definition 1]). The set $\mathcal{C}$ is a polyhedral subdivision if $\mathcal{C}$ is a polyhedral decomposition and the intersection of any two members of $\mathcal{C}$ is either empty or a common proper face of both.

Definition 2.6 (Piecewise Linear-Quadratic (PLQ) function [RW98]). A function $f: \mathbb{R}^{d} \rightarrow \mathbb{R} \cup$ $\{+\infty\}$ is a Piecewise Linear-Quadratic (PLQ) function if its domain can be represented as the union of finitely many polyhedral sets on each of which the function is either linear or quadratic.

The domain of a PLQ function can always be decomposed into a polyhedral decomposition and on each set $C_{k}$, we note $f(x)=f_{k}(x)$ if $x \in C_{k}, k \in \mathcal{K}$, where

$$
f_{k}(x)=\frac{1}{2} x^{T} Q_{k} x+q_{k}^{T} x+\alpha_{k}
$$

with $Q_{k}$ a $d \times d$ symmetric matrix, $q_{k} \in \mathbb{R}^{d}$ and $\alpha_{k} \in \mathbb{R}$. For each piece of the PLQ function $f$, we associate the function $\tilde{f}_{k}=f_{k}+\delta_{C_{k}}$ where $\delta_{C_{k}}$ is the indicator function defined as

$$
\delta_{C_{k}}= \begin{cases}0, & \text { if } x \in C_{k} ; \\ \infty, & \text { if } x \notin C_{k} .\end{cases}
$$

Example 2.7. The $l_{1}$ norm function, illustrated in Figure 1, is a PLQ function that will be used throughout the paper as an example. Its domain is partitioned into four polyhedral sets on each of which the function is linear,

$$
l_{1}\left(x_{1}, x_{2}\right)=\left|x_{1}\right|+\left|x_{2}\right|= \begin{cases}x_{1}+x_{2}, & \text { if }-x_{1} \leq 0,-x_{2} \leq 0 \\ -x_{1}+x_{2}, & \text { if } x_{1} \leq 0,-x_{2} \leq 0 \\ -x_{1}-x_{2}, & \text { if } x_{1} \leq 0, x_{2} \leq 0 \\ x_{1}-x_{2}, & \text { if }-x_{1} \leq 0, x_{2} \leq 0\end{cases}
$$

Throughout this paper we assume that the input function is a proper convex lsc bivariate PLQ function whose domain is a polyhedral subdivision.

Definition 2.8 (Entity [GL13]). Assume $f$ is a PLQ function and $\cup_{k} C_{k}=\operatorname{dom} f$ is a polyhedral subdivision that induces a partition of $\operatorname{dom} f$. An entity is an element of the partition of the domain. For $f: \mathbb{R}^{2} \rightarrow \mathbb{R} \cup\{+\infty\}$, an entity is either a vertex, an edge or a face.

Definition 2.9 (Entity types [Jak13]). If the dimension of an entity is 0 then it is called a vertex.

If the dimension of an entity is 1 then it is called an edge and can be written

$$
\mathcal{E}_{\Lambda}=\left\{x \in \mathbb{R}^{d}: x=\lambda_{1} x_{1}+\lambda_{2} x_{2}, \lambda_{1}+\lambda_{2}=1, \lambda=\left(\lambda_{1}, \lambda_{2}\right) \in \Lambda\right\},
$$

where $x_{1} \neq x_{2}$. In $\mathbb{R}^{2}$, an edge is classified as a 


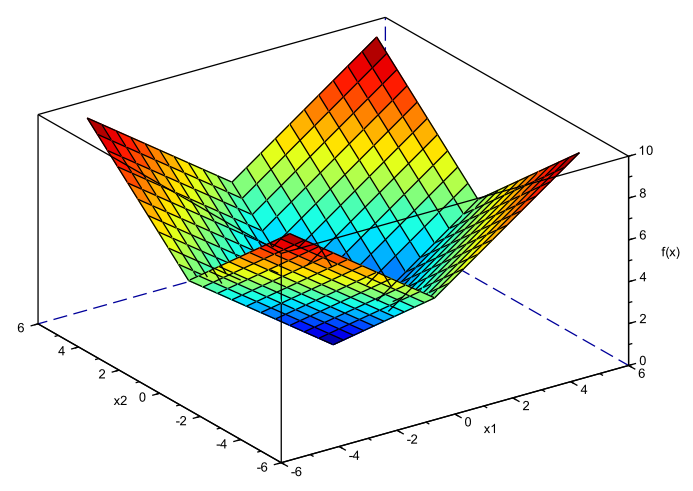

(a)

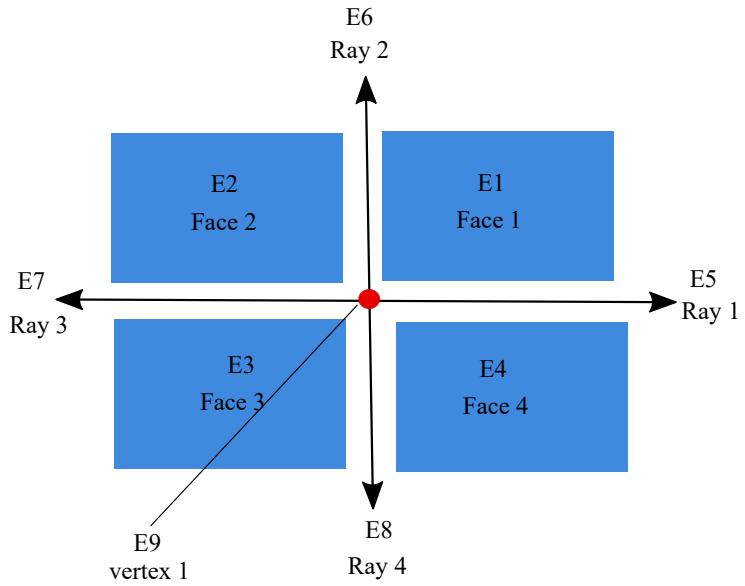

(b)

Figure 1: (a) A bivariate PLQ function - the $l_{1}$ norm function (b) Partition of domain of the $l_{1}$ norm function.

(i) Line when $\Lambda=\mathbb{R}^{2}$.

(ii) Ray when $\Lambda=\mathbb{R}_{+} \times \mathbb{R}$ where $\mathbb{R}_{+}=\{x \in \mathbb{R}: x \geq 0\}$.

(iii) Segment when $\Lambda=\mathbb{R}_{+} \times \mathbb{R}_{+}$.

If an entity has a nonempty interior then it is called a face.

Definition 2.10 (Extreme point, [Fan63]). An extreme point of a convex set $C$, is a point $x \in C$, such that if $x=\theta x_{1}+(1-\theta) x_{2}$ with $x_{1}, x_{2} \in C$ and $\theta \in[0,1]$, then $x_{1}=x$ or $x_{2}=x$ (or both).

Definition 2.11 (Subgradient, subdifferential [Roc70, RW98]). The subgradient of a function $f$ at a point $\bar{x} \in \operatorname{dom} f$, is a vector $s$ such that

$$
f(x) \geq f(\bar{x})+\langle s, x-\bar{x}\rangle, \forall x \in \mathbb{R}^{d},
$$

where $\langle\cdot, \cdot$,$\rangle denotes the standard dot product.$

The subdifferential of a function $f$ at a point $\bar{x}$ is a closed convex set which is the collection of all subgradients of $f$ at $\bar{x}$. It is noted

$$
\partial f(x)=\left\{s \in \mathbb{R}^{d}: f(x) \geq f(\bar{x})+\langle s, x-\bar{x}\rangle, \forall x \in \operatorname{dom} f\right\} .
$$

If $x \notin \operatorname{dom} f$ by convention we set $\partial f(x)=\emptyset$.

A subgradient of the PLQ function $f$, defined by Equation (1), at a point $x$ is

$$
s=\nabla f_{k}(x)=Q_{k} x+q_{k} .
$$

If the function is convex and differentiable at $x \in \operatorname{int} \operatorname{dom} f$, then $\partial f(x)=\{\nabla f(x)\}$. If a PLQ function $f$ is not differentiable at $x$ then its subdifferential is

$$
\partial f(x)=\operatorname{co}\left\{\nabla f_{k}(x): \text { for all } k \text { such that } x \in C_{k}\right\} .
$$


Definition 2.12 (Planar graph). A graph $\mathcal{G}=(\mathcal{V}, \mathcal{E})$ is said to be a planar graph if it can be drawn in a plane with no two edges crossing each other except at a vertex to which they are incident.

The following property states that planar graphs are sparse i.e. they do not have many edges.

Proposition 2.13 ([AMO93, Property 8.7]). Let $\mathcal{G}=(\mathcal{V}, \mathcal{E})$ be a connected planar graph with $n_{e}$ edges and $n_{v}$ vertices. Assume $n_{v} \geq 3$. Then $n_{e} \leq 3 n_{v}$.

We include the proof for completeness. First, we recall Euler's Formula.

Fact 2.14 (Euler's Formula). For a connected planar graph $\mathcal{G}$ with $n_{v}$ vertices, $n_{e}$ edges and $n_{f}$ faces, we have $n_{v}-n_{e}+n_{f}=2$.

Proof. In a planar graph $\mathcal{G}$, the edges divide the plane into different regions and each region is called a face of the graph $\mathcal{G}$. The total number of edges bordering a face $F_{i}$ is called the degree of $F_{i}$. An edge separates 2 faces so $\sum_{i=1}^{m} \operatorname{deg} F_{i}=2 n_{e}$.

Since any face has degree $\operatorname{deg} F_{i} \geq 3$, we obtain $2 n_{e}=\sum_{i=1}^{m} \operatorname{deg} F_{i} \geq 3 n_{f}$; hence $n_{f} \leq \frac{2}{3} n_{e}$. Using Euler's Formula gives $n_{f}=2+n_{e}-n_{v} \leq \frac{2}{3} n_{e} ;$ so $\frac{1}{3} n_{e} \leq n_{v}-2$, and $n_{e} \leq 3 n_{v}-6$.

Since graph-matrix calculus is a fundamental part of our algorithm, we recall the following fact. We note

$$
\operatorname{gph} \partial f=\left\{(x, s) \in \mathbb{R}^{d} \times \mathbb{R}^{d}: s \in \partial f(x)\right\}
$$

the graph of the subdifferential and Id the identity matrix.

Fact 2.15 (Goebel's Graph-matrix calculus [Goe08, GL11]). Assume $f: \mathbb{R}^{d} \rightarrow \mathbb{R} \cup\{+\infty\}$ is a proper lsc convex function, then

$$
\operatorname{gph} \partial\left(f^{*}\right)=\left[\begin{array}{cc}
0 & \mathrm{Id} \\
\mathrm{Id} & 0
\end{array}\right] \operatorname{gph} \partial f
$$

In other words, one can compute the graph of the subdifferential of the conjugate by applying a linear transform to the graph of the subdifferential of the function. We will use the result to associate a dual point $\left(x, s, y^{*}\right)$ to any primal point $(x, s, y)$ with $y^{*}=s^{T} x-y$. Note that the result is vectorized, i.e. a program like MATLAB can compute the result using vector operations resulting in increased efficiency on any modern computer.

\section{Representation of a PLQ function}

The input for our algorithm is a graph, called the entity graph, that stores specific information on each entity. The entity graph is built from a proper convex lsc PLQ function. Each node of the entity graph represents an entity of the PLQ function.

Let $\mathcal{G}=(\mathcal{V}, \mathcal{E})$ be the entity graph where $\mathcal{V}$ is the set of nodes and $\mathcal{E}$ is the set of edges. Each node represents an entity. When two entities are adjacent, we connect them using an edge. For example, the domain of the $l_{1}$ norm function is partitioned into four faces, four rays and one vertex, which is illustrated in Figure 1 while Figure 2 is the corresponding entity graph. In addition, each node of $\mathcal{G}$ stores the following information about its entity: the GPH matrix, the adjacent entities and the entity type. 


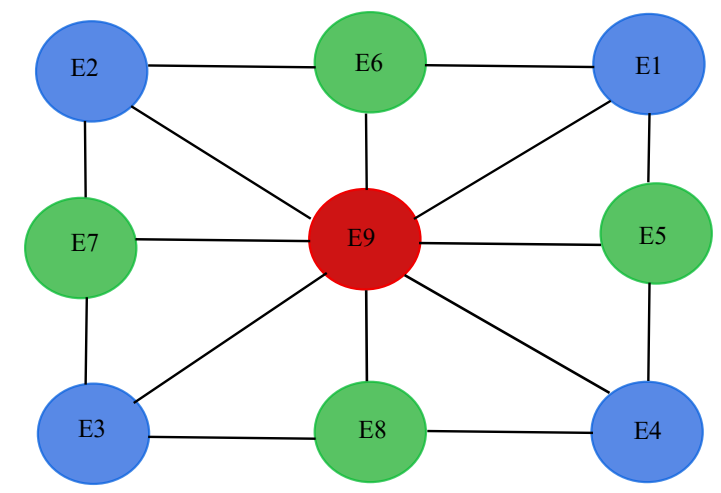

Figure 2: Entity graph for the $l_{1}$ norm function

\subsection{GPH matrix}

We use a GPH matrix to represent an entity $C_{k}$ and its associated function $f_{k}$ [Luc13b]. For each entity $k$, we select some points from $C_{k}$ and compute the full subdifferential and the function value at those points. Points in the GPH matrix representation are stored in order. Suppose we pick $n$ points to completely represent $C_{k}$. Its GPH matrix $G_{k}$ is

$$
G_{k}=\left[\begin{array}{cccc}
x_{1} & x_{2} & \ldots & x_{n} \\
s_{1} & s_{2} & \ldots & s_{n} \\
y_{1} & y_{2} & \ldots & y_{n} \\
b_{1} & b_{2} & \ldots & b_{n} \\
b_{1}^{*} & b_{2}^{*} & \ldots & b_{n}^{*}
\end{array}\right]
$$

where $x_{i} \in C_{k}$ is the coordinate of a point, $s_{i}$ is a subgradient of $f$ at the point $x_{i}$, and $y_{i}$ is the value of $f$ at $x_{i}$. The points $x_{i}$ represent the polyhedral set $C_{k}$ and are given in clockwise order. Similarly, the points $s_{i}$ describe the full subgradient $\partial f\left(x_{i}\right)$, which is a polyhedral set. The binary flag $b_{i}$ (resp. $b_{i}^{*}$ ) is used to identify whether $x_{i}$ (resp. $s_{i}$ ) is an extreme point. It equals 0 for an extreme point and 1 otherwise.

In $\mathbb{R}^{d}$, the dimension of the GPH matrix is $(2 d+3) n$, where $n$ is the number of points. For example, in $\mathbb{R}^{2}$, the dimension of a GPH matrix is $7 n$.

Example 3.1. Consider $f\left(x_{1}, x_{2}\right)=\left(x_{1}^{2}+x_{2}^{2}\right) / 2$ if $x_{1} \geq 0, x_{2} \geq 0$ and $\infty$ otherwise, whose conjugate is

$$
f^{*}\left(s_{1}, s_{2}\right)= \begin{cases}\frac{1}{2}\left(s_{1}^{2}+s_{2}^{2}\right), & \text { if } s_{1} \geq 0, s_{2} \geq 0 \\ \frac{1}{2} s_{2}^{2}, & \text { if } s_{1} \leq 0, s_{2} \geq 0 \\ 0, & \text { if } s_{1} \leq 0, s_{2} \leq 0 \\ \frac{1}{2} s_{1}^{2}, & \text { if } s_{1} \geq 0, s_{2} \leq 0\end{cases}
$$

Hence, the domain of the conjugate is composed of 9 entities: 4 faces, 4 rays, and 1 vertex. The domain of the PLQ function $f$ and the domain of its conjugate $f^{*}$ are shown in Figure 3.

To compute the GPH matrix for the entity $E_{1}=\left\{\left(x_{1}, 0\right): x_{1} \geq 0\right\}$, a ray, we note that $E_{1}$ is adjacent to $F_{1}=\left\{x: x_{1}, x_{2} \geq 0\right\}$ (associated with $f_{1}\left(x_{1}, x_{2}\right)=\frac{1}{2} x_{1}^{2}+\frac{1}{2} x_{2}^{2}$ ) and entity $F_{2}=\left\{x: x_{1} \geq 0, x_{2} \leq 0\right\}$ (associated with $\left.f_{2}\left(x_{1}, x_{2}\right)=\infty\right)$. Since $\operatorname{dom} f_{2}=\emptyset, \partial f_{2}\left(x_{1}, x_{2}\right)=\emptyset$.

We note that for any point $x \in E_{1}, \partial \tilde{f}_{1}(x)=\{0\} \times(-\infty, 0]$. Hence, we need 2 points to store $E_{1}$ (we have to select $(0,0)$ since it is an extreme point and we arbitrarily pick $(1,0)$ to represent the 

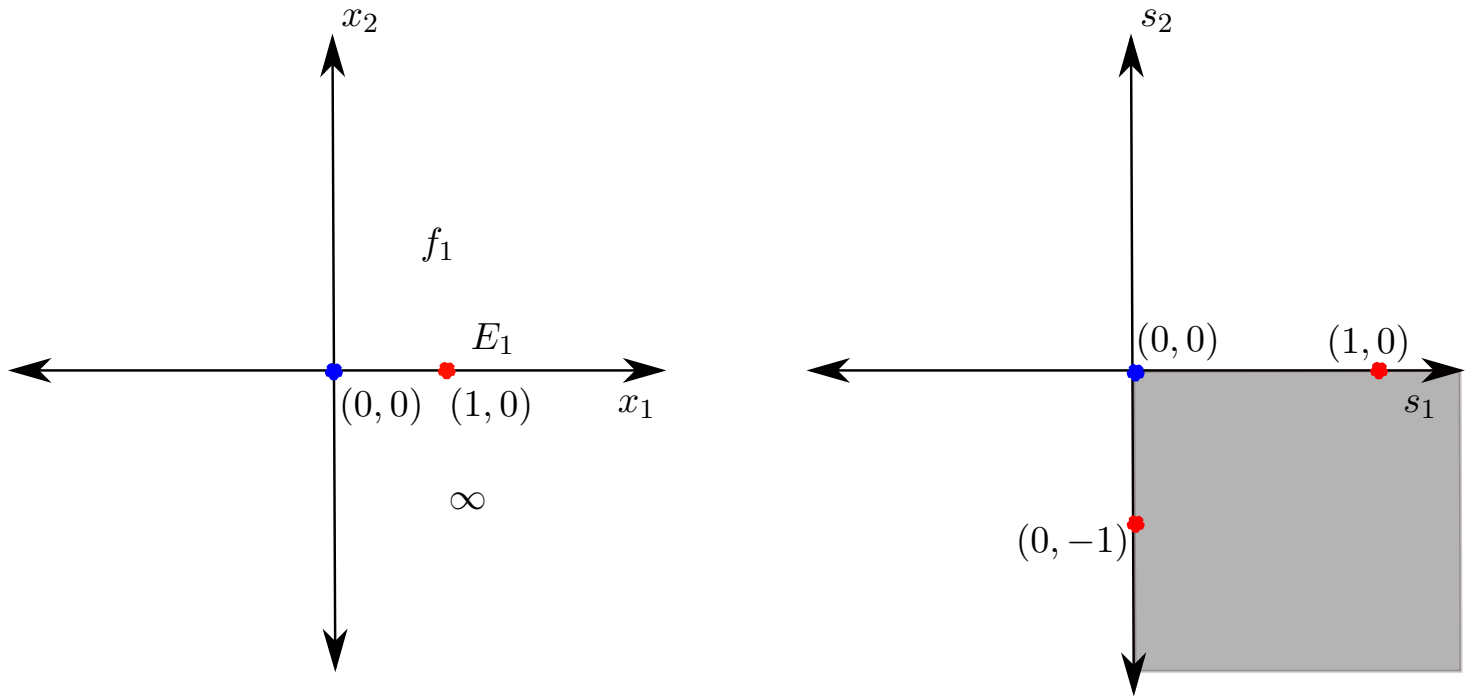

(a) Primal graph. The ray $E_{1}=\left\{\left(x_{1}, 0\right): x_{1} \geq 0\right\}$ is (b) Dual graph. The gray area represents $\partial f\left(E_{1}\right)=$ represented using extreme point $(0,0)$ and the arbitrar- $\cup_{x \in E_{1}} \partial f(x)$ where $\partial f(x)=\{0\} \times(-\infty, 0]$; it is repreily chosen nonextreme point $(1,0)$. It separates region sented using the extreme point $(0,0)$ and the (arbitrarily $F_{1}$ associated with function $f_{1}$ from region $F_{2}$ associated chosen) nonextreme points $(1,0)$ and $(0,-1)$. with $f_{2}=+\infty$.

Figure 3: The domain of Function $f$ from Example 3.1 (left) and the domain of its conjugate (right). Blue dots represent extreme points while red dots represent non-extreme points.

direction) but 3 points to store its associated entity $\mathbb{R}^{+} \times \mathbb{R}^{-}$(again we have to select the extreme point $(0,0)$ and arbitrarily pick $(0,-1)$ and $(1,0)$ to represent the 2 directions). Consequently, $a$ GPH matrix $G_{1}$ for $E_{1}$ is

$$
G_{1}=\left[\begin{array}{ccc}
1 & 0 & 0 \\
0 & 0 & 0 \\
1 & 0 & 0 \\
0 & 0 & -1 \\
1 / 2 & 0 & 0 \\
1 & 0 & 0 \\
1 & 0 & 1
\end{array}\right]
$$

We note that storing extreme points and directions is sufficient to represent any polyhedral set.

Fact 3.2. (Representation of a polyhedral set [Roc70, Theorem 19.1, Corollary 19.1.1]) Assume $C=\left\{x \in \mathbb{R}^{2}: a_{i}^{T} x \leq b_{i}, i=1, \ldots, m\right\}$ is a polyhedral set with at least one extreme point. Then the set of extreme points of $C$ contains a finite number of elements $x_{1}, x_{2}, \cdots, x_{k}$. If $C$ is bounded then the set of extreme direction is empty. If $C$ is not bounded then the set of extreme directions is nonempty and has a finite number of elements $d_{1}, d_{2}, \ldots, d_{l}$. Moreover, $\bar{x} \in C$ if and only if

$$
\bar{x}=\sum_{i=1}^{k} \lambda_{i} x_{i}+\sum_{j=1}^{l} \mu_{j} d_{j},
$$

where $\sum_{i=1}^{k} \lambda_{i}=1, \lambda_{i} \geq 0, i=1, \ldots, k, \mu_{j} \geq 0, j=1, \ldots, l$. 
After we compute the conjugate $f^{*}$ and provided it with our data structure, the user may wish to compute the quadratic formula associated with each entity; such computation can be performed by interpolation as follow. Given the GPH matrix $G_{k}$, we compute the entity $C_{k}$ and the associated function $f_{k}$ by solving a linear system amounting to Hermite interpolation. Recall that, on each piece of the domain the function $f$ is quadratic and represented by Equation (1). The subgradient of $f$ at a point $x$ is represented by Equation (2). For each $x$ we get $d$ equations from (2) (gradient information) and 1 equation from knowing the function value. So for each $x$ we have a total of $d+1$ equations.

The number of unknowns to determine the symmetric matrix $Q_{k}$ is $d+d-1+\cdots+1=d(d+1) / 2$, while it is $d$ for $q_{k}$ and 1 for $\alpha_{k}$ giving a total of $d(d+1) / 2+d+1=(d+1) d / 2+1$ unknowns. Consequently, the minimum number of points required to determine the function $f_{k}$ is $(\lceil d / 2\rceil+1)$.

In $\mathbb{R}^{2}$, we need to determine 6 unknowns. For each point we get 3 equations. So we need to store at least two points to determine $f_{k}$ uniquely, although we may need to store more to represent $C_{k}$.

Example 3.3. Note

$$
f_{k}(x)=y=\frac{1}{2}\left[\begin{array}{ll}
x_{1} & x_{2}
\end{array}\right]\left[\begin{array}{ll}
a & b \\
b & c
\end{array}\right]\left[\begin{array}{l}
x_{1} \\
x_{2}
\end{array}\right]+\left[\begin{array}{ll}
q_{1} & q_{2}
\end{array}\right]\left[\begin{array}{l}
x_{1} \\
x_{2}
\end{array}\right]+\alpha
$$

Equation (2) gives

$$
\begin{aligned}
& s_{1}=a x_{1}+b x_{2}+q_{1}, \\
& s_{2}=b x_{1}+c x_{2}+q_{2} .
\end{aligned}
$$

Consider the entity $E_{1}$ of Example 2.7, which is a face. Its GPH matrix $G_{1}$ is

$$
G_{1}=\left[\begin{array}{lll}
1 & 0 & 0 \\
0 & 0 & 1 \\
1 & 1 & 1 \\
1 & 1 & 1 \\
1 & 0 & 1 \\
1 & 0 & 1 \\
0 & 0 & 0
\end{array}\right] .
$$

We need 3 points to represent $E_{1}$. The matrix $G_{1}$ indicates that $(0,0)$ is an extreme point while $(1,0)$ and $(0,1)$ are non extreme points.

To determine $f_{1}$, the function associated with $E_{1}$, we need to compute $\left(a, b, c, q_{1}, q_{2}, \alpha\right)$. From Equation (3) we solve

$$
\left[\begin{array}{l}
1 \\
1 \\
1
\end{array}\right]=\left[\begin{array}{lll}
1 & 0 & 1 \\
0 & 0 & 1 \\
0 & 1 & 1
\end{array}\right]\left[\begin{array}{c}
a \\
b \\
q_{1}
\end{array}\right] .
$$

to obtain $\left(a, b, q_{1}\right)=(0,0,1)$. Similarly using (4) we compute $\left(b, c, q_{2}\right)=(0,0,1)$ and substituting we get $\alpha=0$. We deduce $f_{1}\left(x_{1}, x_{2}\right)=x_{1}+x_{2}$.

In the GPH matrix representation, we store a point $x$ multiple times when its subdifferential is multi-valued [GL11, GL10].

Example 3.4. Consider the entity $E_{5}$ of Example 2.\%. It is a ray adjacent to two faces: $E_{1}$ and $E_{4}$. Consider $x=(0,0)$. The face $E_{1}$ is associated with $f_{1}(x)=x_{1}+x_{2}$ and the only subgradient of $f_{1}$ at $x=(0,0)$ is $(1,1)$. Similarly, the face $E_{4}$ is associated with $f_{4}(x)=x_{1}-x_{2}$, and at 
$x=(0,0)$, the subgradient is $(1,-1)$. At any point on $E_{5}$ (except $(0,0)$ ) the subdifferential is $\partial f\left(x_{1}, x_{2}\right)=\{1\} \times[1,-1]=\operatorname{co}\{(1,1),(1,-1)\}$. It has two extreme points $(1,1)$ and $(1,-1)$. We represents the $G P H$ matrix $G_{5}$ associated with $E_{5}$ as

$$
G_{5}=\left[\begin{array}{cccc}
1 & 0 & 0 & 1 \\
0 & 0 & 0 & 0 \\
1 & 1 & 1 & 1 \\
1 & 1 & -1 & -1 \\
1 & 0 & 0 & 1 \\
1 & 0 & 0 & 1 \\
0 & 0 & 0 & 0
\end{array}\right]
$$

Remark 3.5. In $\mathbb{R}^{2}$, we can recover the polyhedral set by computing the convex hull of $x_{i}$ where $i=1, \cdots, n$ ( $s_{i}$ in the dual). Note that we need at least one extreme point to recover the polyhedral set, so we may split $\operatorname{dom} f$ further to make sure any entity $C_{k}$ has at least one extreme point.

For our algorithm, we choose to store the extreme points in order as we move along the convex hull in clockwise fashion. Since there are either 0 (bounded set), 1 (ray), or 2 (face) directions, we store nonextreme points for unbounded sets as the first and last column.

In our Scilab implementation, we use two hypermatrices $H_{p}$ and $H_{d}$ to store the GPH matrices of all entities in the domain of $f$ and the domain of $f^{*}$ respectively. In $\mathbb{R}^{d}$, the dimension of the hypermatrix is $N \times M \times n_{\max }$ where $N$ is the total number of entities, $M=(2 d+3)$ is the number of rows of the GPH matrix and $n_{\max }=\max \left\{n_{i}: i=1, \ldots, N\right\}$ where $n_{i}$ is the number of columns in the GPH matrix of entity $i$.

Example 3.6. The function $l_{1}$ norm has nine entities and the maximum value of $n_{i}$ is 4 which is attained for the vertex $E_{9}$. So the dimension of the hypermatrix $H_{p}$ used to store the entities of the $l_{1}$ norm is $9 \times 7 \times 4$.

\subsection{Adjacent entities}

Each node of $\mathcal{G}$ stores its adjacent entities information in a sparse $N \times m$ matrix, called the neighbor matrix, where $\mathrm{N}$ is the number of entities and $m$ is the maximum degree of all vertices in the entity graph.

Example 3.7. Consider Example 2.7, which has 9 entities. The vertex $E_{9}$ has eight adjacent entities which is the maximum. So we use a Neighbor Matrix of dimension $9 \times 8$. The entity $E_{1}$ is adjacent to $E_{5}, E_{6}$ and $E_{9}$. In the entity graph $\mathcal{G}$, the node $\mathcal{V}_{1}$ which contains $E_{1}$, stores the indices of the adjacent entities i.e. 5, 6, 9. We represent the Neighbor Matrix of Example 2.7 as

$$
N_{M}=\left[\begin{array}{llllllll}
5 & 6 & 9 & 0 & 0 & 0 & 0 & 0 \\
6 & 7 & 9 & 0 & 0 & 0 & 0 & 0 \\
7 & 8 & 9 & 0 & 0 & 0 & 0 & 0 \\
5 & 8 & 9 & 0 & 0 & 0 & 0 & 0 \\
1 & 4 & 9 & 0 & 0 & 0 & 0 & 0 \\
1 & 2 & 9 & 0 & 0 & 0 & 0 & 0 \\
2 & 3 & 9 & 0 & 0 & 0 & 0 & 0 \\
3 & 4 & 9 & 0 & 0 & 0 & 0 & 0 \\
1 & 2 & 3 & 4 & 5 & 6 & 7 & 8
\end{array}\right]
$$

Each row of this matrix contains the adjacency information of the corresponding entity. For example, the index of all adjacent entities of $E_{5}$ is found in the $5^{\text {th }}$ row of $N_{M}$. 


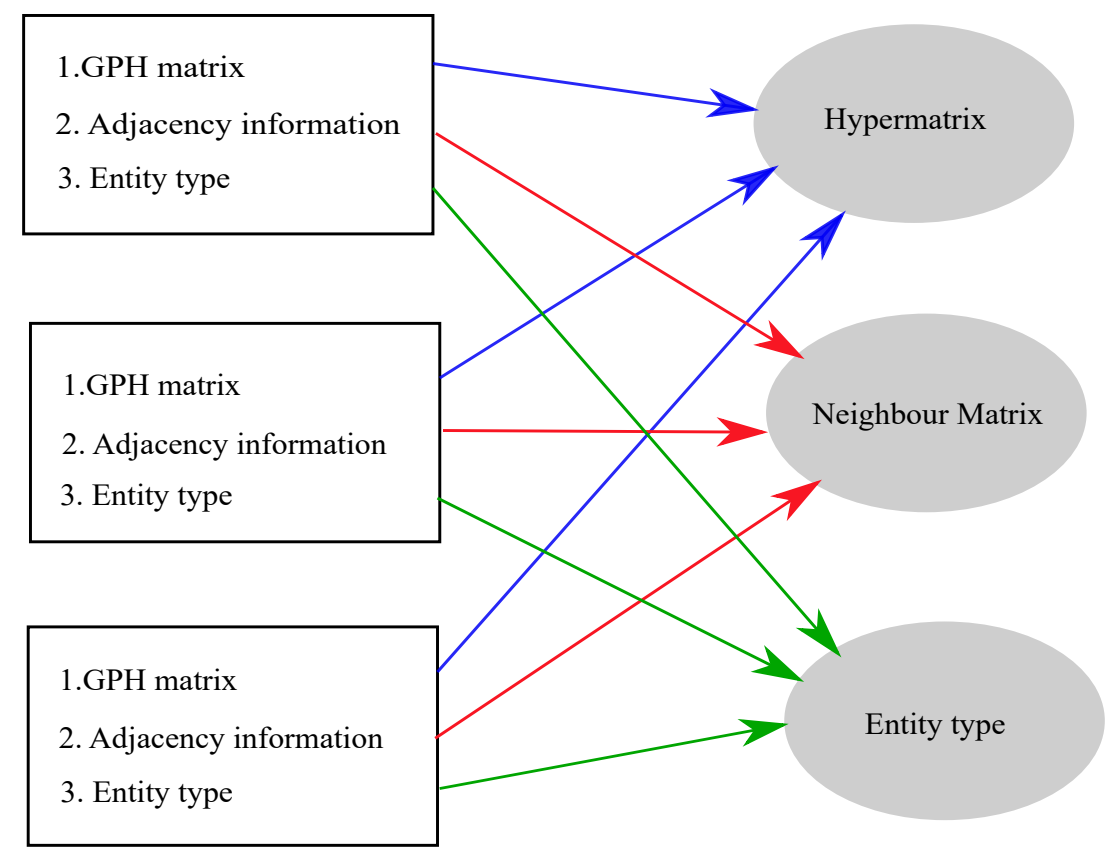

Figure 4: Visualization of the relationship between the information stored for each entity and the data structure recording that information.

\subsection{Entity Type}

The type of each entity is stored as a single integer whose meaning is listed in Table 2. We use an $1 \times N$ array $T$ to store the type of all entities.

Table 2: Entity type.

\begin{tabular}{c|c}
\hline Entity Type & Flag \\
\hline Vertex & 1 \\
Face & 2 \\
Line & 3 \\
Ray & 4 \\
Segment & 5 \\
\hline
\end{tabular}

Example 3.8. The array which stores the entity type of the $l_{1}$ norm function is

$$
T=\left[\begin{array}{lllllllll}
2 & 2 & 2 & 2 & 4 & 4 & 4 & 4 & 1
\end{array}\right] .
$$

It indicates that entities $E_{1}$ to $E_{4}$ are faces, entities $E_{5}$ to $E_{8}$ are rays and $E_{9}$ is a vertex.

We visualize our data structure in Figure 4. Consider a PLQ function with $N$ entities where each entity has a GPH matrix, adjacency information and entity type. We store all GPH matrices in a hypermatrix, all adjacency information in a neighbor matrix $N_{M}$ and all entity types in an array $T$. 


\section{Algorithm for computing the conjugate of a PLQ function}

Consider $f: \mathbb{R}^{2} \rightarrow \mathbb{R} \cup\{+\infty\}$ where $\operatorname{dom} f$ has $N$ entities. Algorithm 1 uses breadth-first search to traverse the entity graph $\mathcal{G}$. When Entity $i$ is considered, we store the index of all adjacent entities in an array denoted $D$ of dimension $1 \times N$. We traverse $\mathcal{G}$ according to the index stored in $D$. Note that we will not store any duplicate index in $D$. To check whether the index of an entity is already stored in $D$, we use a binary array $I$ of dimension $1 \times N\left(I_{i}=1\right.$ if entity $i$ is already stored in $D$, otherwise $I_{i}=0$ ).

Algorithm 1 calls two subroutines: Algorithm 2 and Algorithm 3. Algorithm 2 computes the dual entity $G_{d}$ and type $t$ of the primal entity $G_{p}$. It uses graph-matrix calculus on Line 6 to obtain $G_{d}$ while the remainder of the algorithm computes $t$. Algorithm 3 updates $D, I, \bar{N}$ using information from $E_{a d j}$. It uses the binary array $I$ to store unique indices in $D$ and allows the main loop of Algorithm 1 to pick the next non-visited index at Line 5 in Algorithm 1.

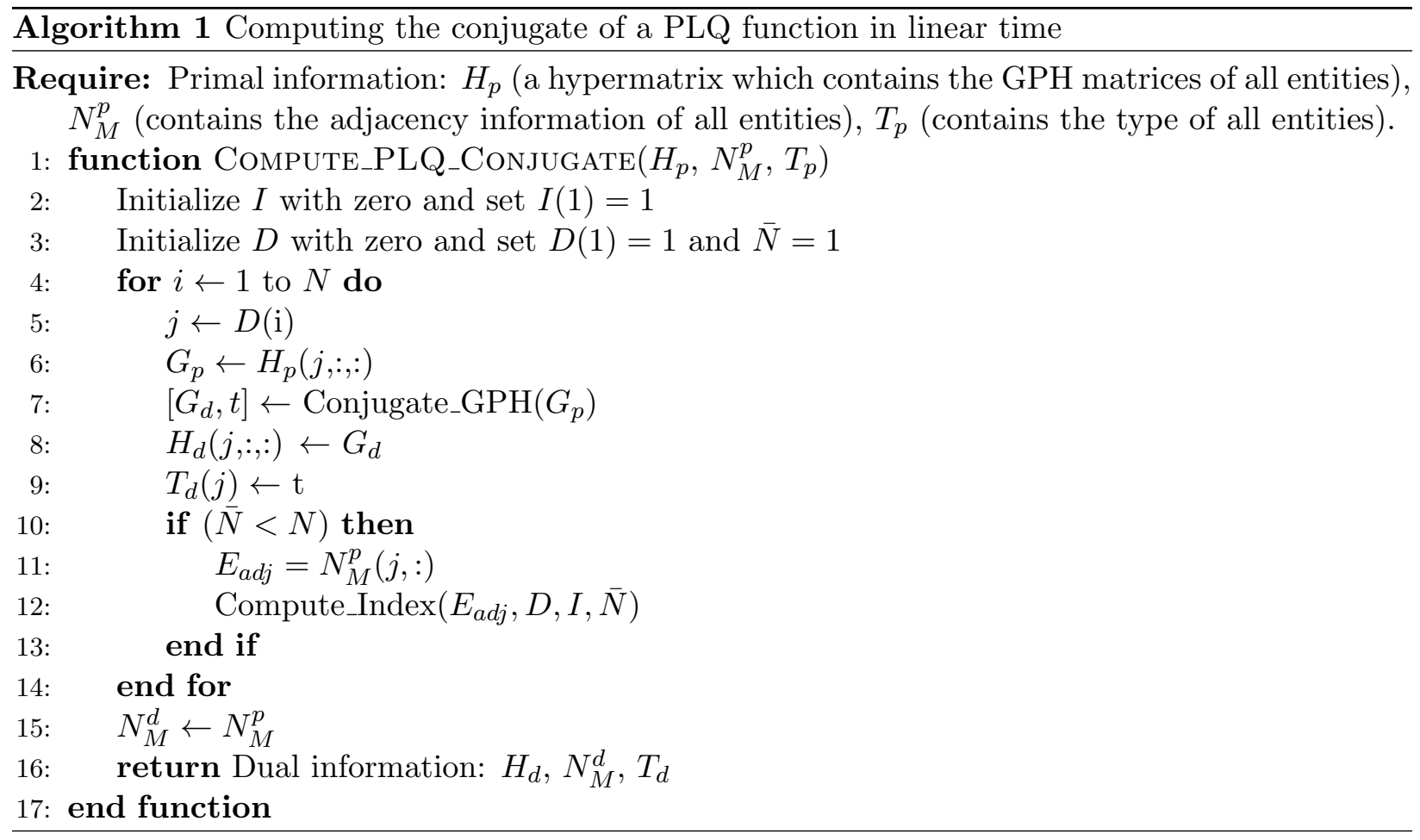

Example 4.1. Applying Algorithm 1 to the $l_{1}$ norm function whose entity graph is illustrated on Figure 2, we start from the face $E_{1}$ with GPH matrix

$$
G_{1}^{p}=\left[\begin{array}{lll}
1 & 0 & 0 \\
0 & 0 & 1 \\
1 & 1 & 1 \\
1 & 1 & 1 \\
1 & 0 & 1 \\
1 & 0 & 1 \\
0 & 0 & 0
\end{array}\right],
$$

and adjacent entities 5,6,9. We initialize $D=[1,0,0,0,0,0,0,0]$ and $I=[1,0,0,0,0,0,0,0]$, and 


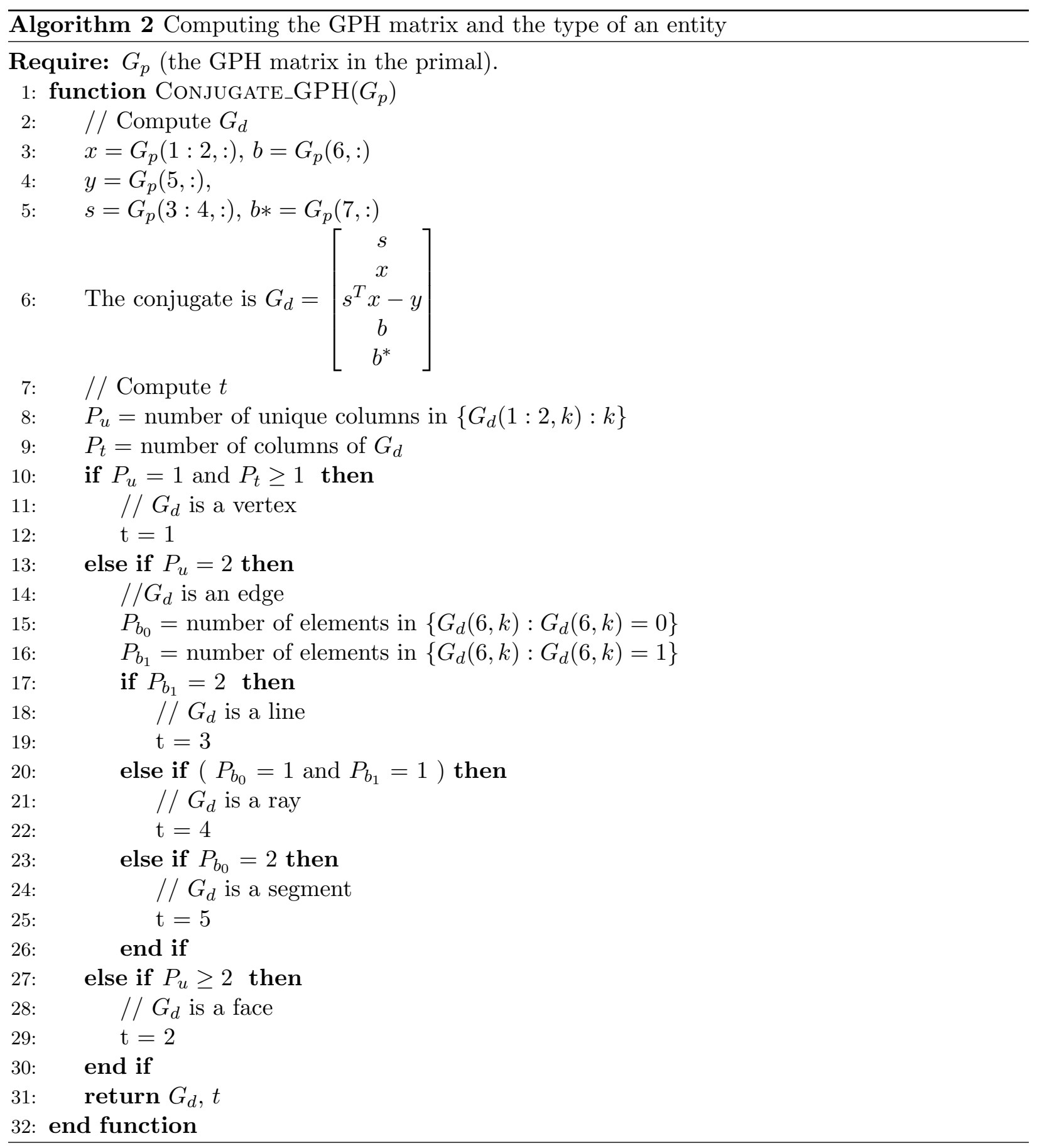


$\bar{N}=1$. We apply Algorithm 2 to compute the conjugate of $E_{1}$ and obtain

$$
G_{1}^{d}=\left[\begin{array}{lll}
1 & 1 & 1 \\
1 & 1 & 1 \\
1 & 0 & 0 \\
0 & 0 & 1 \\
0 & 0 & 0 \\
0 & 0 & 0 \\
1 & 0 & 1
\end{array}\right] .
$$

Since $G_{1}^{d}$ has a unique subgradient, we deduce it is a vertex and set its type to 1.

Next we check the adjacent entities of $E_{1}$ using Algorithm 3.

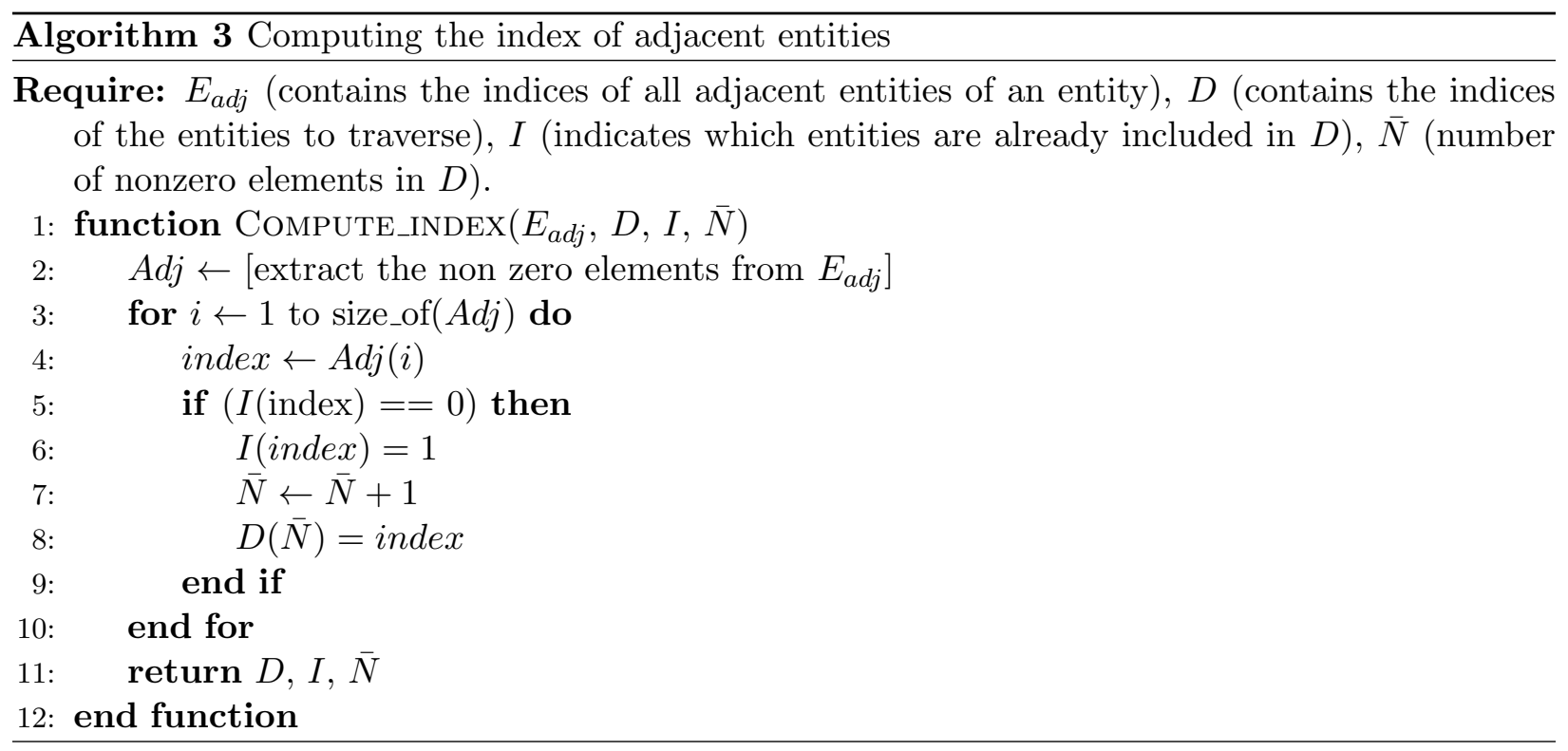

We find that the entity $E_{1}$ is adjacent to the entities $E_{5}, E_{6}$, and $E_{9}$. Then we check the corresponding index of I and see that these entities have not been included in $D$ yet. So we update $D, I$ and $\bar{N}$ as

$$
\begin{aligned}
I & =\left[\begin{array}{lllllllll}
1 & 0 & 0 & 0 & 1 & 1 & 0 & 0 & 1
\end{array}\right], \\
D & =\left[\begin{array}{lllllllll}
1 & 5 & 6 & 9 & 0 & 0 & 0 & 0 & 0
\end{array}\right], \\
\bar{N} & =4
\end{aligned}
$$

and loop to the next entity indicated in D, in this case Entity 5.

We traverse all nodes of $\mathcal{G}$ and update the array $D$ and $I$ accordingly, see Table 3.

Figure 5 shows the partition of the primal and the dual domain of the $l_{1}$ norm function. The mapping of the entities from the primal to the dual domain is presented in Table 4.

\section{Complexity analysis}

The space and time complexity of our algorithm is computed next. 
Table 3: Iterations of Algorithm 1.

\begin{tabular}{c|ccccccccc|ccccccccc}
\hline Iterations & \multicolumn{10}{|c|}{$D$} & & \multicolumn{10}{c}{} & & & & & & & & & \\
\hline Initialization & 1 & 0 & 0 & 0 & 0 & 0 & 0 & 0 & 0 & 1 & 0 & 0 & 0 & 0 & 0 & 0 & 0 & 0 \\
\hline 1 & 1 & 5 & 6 & 9 & 0 & 0 & 0 & 0 & 0 & 1 & 0 & 0 & 0 & 1 & 1 & 0 & 0 & 1 \\
\hline 2 & 1 & 5 & 6 & 9 & 4 & 0 & 0 & 0 & 0 & 1 & 0 & 0 & 1 & 1 & 1 & 0 & 0 & 1 \\
\hline 3 & 1 & 5 & 6 & 9 & 4 & 2 & 0 & 0 & 0 & 1 & 1 & 0 & 1 & 1 & 1 & 0 & 0 & 1 \\
\hline 4 & 1 & 5 & 6 & 9 & 4 & 2 & 3 & 7 & 8 & 1 & 1 & 1 & 1 & 1 & 1 & 1 & 1 & 1 \\
\hline 5 & 1 & 5 & 6 & 9 & 4 & 2 & 3 & 7 & 8 & 1 & 1 & 1 & 1 & 1 & 1 & 1 & 1 & 1 \\
\hline 6 & 1 & 5 & 6 & 9 & 4 & 2 & 3 & 7 & 8 & 1 & 1 & 1 & 1 & 1 & 1 & 1 & 1 & 1 \\
\hline 7 & 1 & 5 & 6 & 9 & 4 & 2 & 3 & 7 & 8 & 1 & 1 & 1 & 1 & 1 & 1 & 1 & 1 & 1 \\
\hline 8 & 1 & 5 & 6 & 9 & 4 & 2 & 3 & 7 & 8 & 1 & 1 & 1 & 1 & 1 & 1 & 1 & 1 & 1 \\
\hline 9 & 1 & 5 & 6 & 9 & 4 & 2 & 3 & 7 & 8 & 1 & 1 & 1 & 1 & 1 & 1 & 1 & 1 & 1 \\
\hline
\end{tabular}

Table 4: Mapping of the primal entity to the dual entity for the $l_{1}$ norm function.

\begin{tabular}{c|l|c|l}
\hline Primal entity & Type & Dual entity & Type \\
\hline$E_{1}$ & face 1 & $E_{1}{ }^{\prime}$ & vertex 1 \\
\hline$E_{2}$ & face 2 & $E_{2}{ }^{\prime}$ & vertex 2 \\
\hline$E_{3}$ & face 3 & $E_{3}{ }^{\prime}$ & vertex 3 \\
\hline$E_{4}$ & face 4 & $E_{4}{ }^{\prime}$ & vertex 4 \\
\hline$E_{5}$ & ray 1 & $E_{5}{ }^{\prime}$ & segment 1 \\
\hline$E_{6}$ & ray 2 & $E_{6}{ }^{\prime}$ & segment 2 \\
\hline$E_{7}$ & ray 3 & $E_{7}{ }^{\prime}$ & segment 3 \\
\hline$E_{8}$ & ray 4 & $E_{8}{ }^{\prime}$ & segment 4 \\
\hline$E_{9}$ & vertex 1 & $E_{9}{ }^{\prime}$ & face 1 \\
\hline & \multicolumn{3}{|l}{}
\end{tabular}

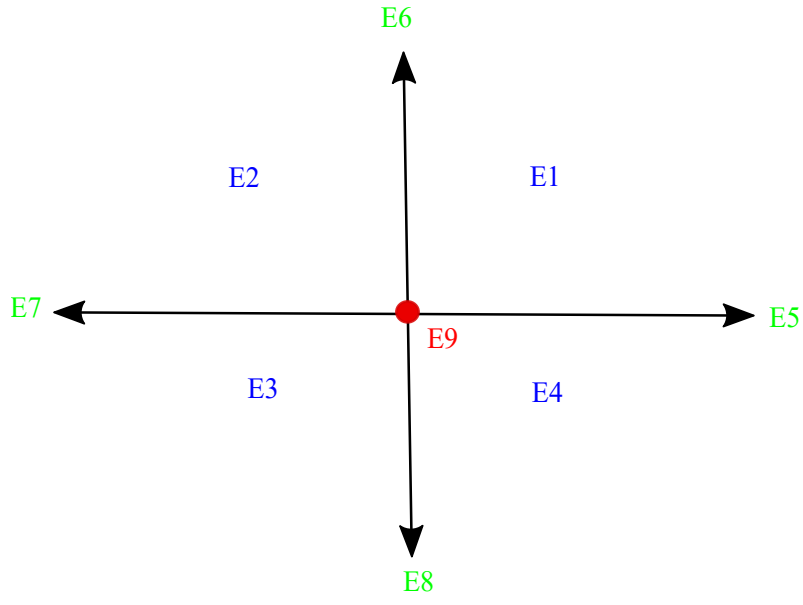

(a)

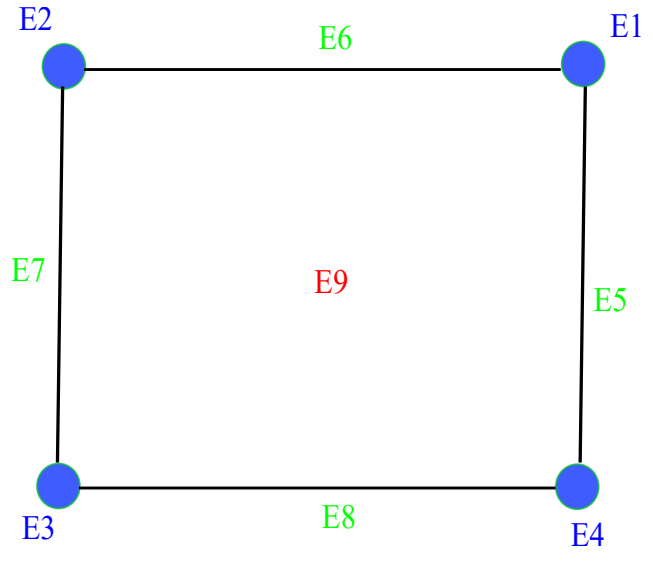

(b)

Figure 5: (a) Partition of $\operatorname{dom} f$ (b) Partition of $\operatorname{dom} f^{*}$. 


\subsection{Space complexity}

Proposition 5.1. Consider a proper convex lsc bivariate PLQ function $f: \mathbb{R}^{2} \rightarrow \mathbb{R} \cup\{+\infty\}$ with $N$ entities. The worst-case space complexity for computing the conjugate of $f$ using Algorithm 1 is $\mathcal{O}\left(N^{2}\right)$ but can be improved to $\mathcal{O}(N)$ using a sparse matrix data structure.

Proof. In Algorithm 1, we use two hypermatrices $\left(H_{p}\right.$ and $\left.H_{d}\right)$ to store the input and output GPH matrices, a Neighbor matrix $N_{M}$ to store the adjacency information, two arrays $\left(T_{p}\right.$ and $\left.T_{d}\right)$ to store the type of entities, an array $D$ to store the indices to traverse and a binary array $I$.

The space complexity of our algorithm mainly depends on the size of the hypermatrix and the Neighbor matrix. Recall that the size of the hypermatrix $H$ is $N \times M \times n_{\max }$ where $\mathrm{N}$ is the total number of entities, $M=(2 d+3)$ is the number of rows of the GPH matrix and $n_{\max }$ is the maximum number of columns in the GPH matrix. If we assume $d$ is constant, the worst-case space complexity of $H$ is $\mathcal{O}(N)$.

In addition, we use four arrays: $T_{p}, T_{d}, D$ and $I$. In the worst-case, the space required to store each array is $\mathcal{O}(N)$.

In Algorithm 1, we use a planar graph as the entity graph. Recall that the sum of the degrees of the vertices equals twice the number of edges and the dimension of the Neighbor matrix $N_{M}$ is $N \times m$ where $m$ is the maximum degree of all vertices. For $N$ entities, in the worst-case the maximum degree of the vertices is $(N-1)$. So the worst-case space complexity of $N_{M}$ is $\mathcal{O}\left(N^{2}\right)$.

Finally, using a sparse matrix data structure we only store the nonzero elements of $N_{M}$, which according to Proposition 2.13, amounts to $\mathcal{O}(N)$.

Remark 5.2. An adjacency list data structure would also result in a $\mathcal{O}(N)$ space complexity; the quadratic space complexity of our implementation is a Scilab limitation.

\subsection{Time complexity}

Proposition 5.3. The worst-case time complexity for computing the conjugate of a proper convex lsc bivariate PLQ function $f$ with $N$ entities using Algorithm 1 is $\mathcal{O}(N)$.

Proof. The time complexity of our algorithm comes from the following parts

(i) Extracting $N$ GPH matrices from the hypermatrix $H_{p}$ according to the index stored in $D$ and storing them in $E$,

(ii) Computing the conjugate of $E$,

(iii) Checking the adjacency information of $E$ and updating the arrays $D$ and $I$,

(iv) Storing the GPH matrix containing the conjugate of $E$ in $H_{d}$.

Task (i) amounts to accessing an index in hypermatrix $H_{p}$, which takes $\mathcal{O}(1)$; the total time required to access $N$ elements of $H_{p}$ is $\mathcal{O}(N)$. Task (ii) involves computing the conjugate and takes $\mathcal{O}(N)$. Task (iii) involves storing the index of adjacent entities in the array $D$. Recall that the indices stored in $D$ are used to traverse the entity graph. In this part, when storing an index in $D$ we need to check whether the index is already stored in $D$ or not. In the worse-case, searching the index of an entity in an array takes $\mathcal{O}(N)$ time. For $N$ entities, the time for storing all indices in $D$ would then be $\mathcal{O}\left(N^{2}\right)$. However, we use a binary array $I$ to determine whether an index is already stored in $D$. Accessing a position of $I$ takes constant time; so the time complexity for $N$ entities reduces to $\mathcal{O}(N)$. Finally, task (iv) requires accessing a position of hypermatrix $H_{d}$ and storing the output GPH matrix in $H_{d}$, which is performed in linear time like Task (i). Consequently, the overall time complexity for Algorithm 1 is linear. 


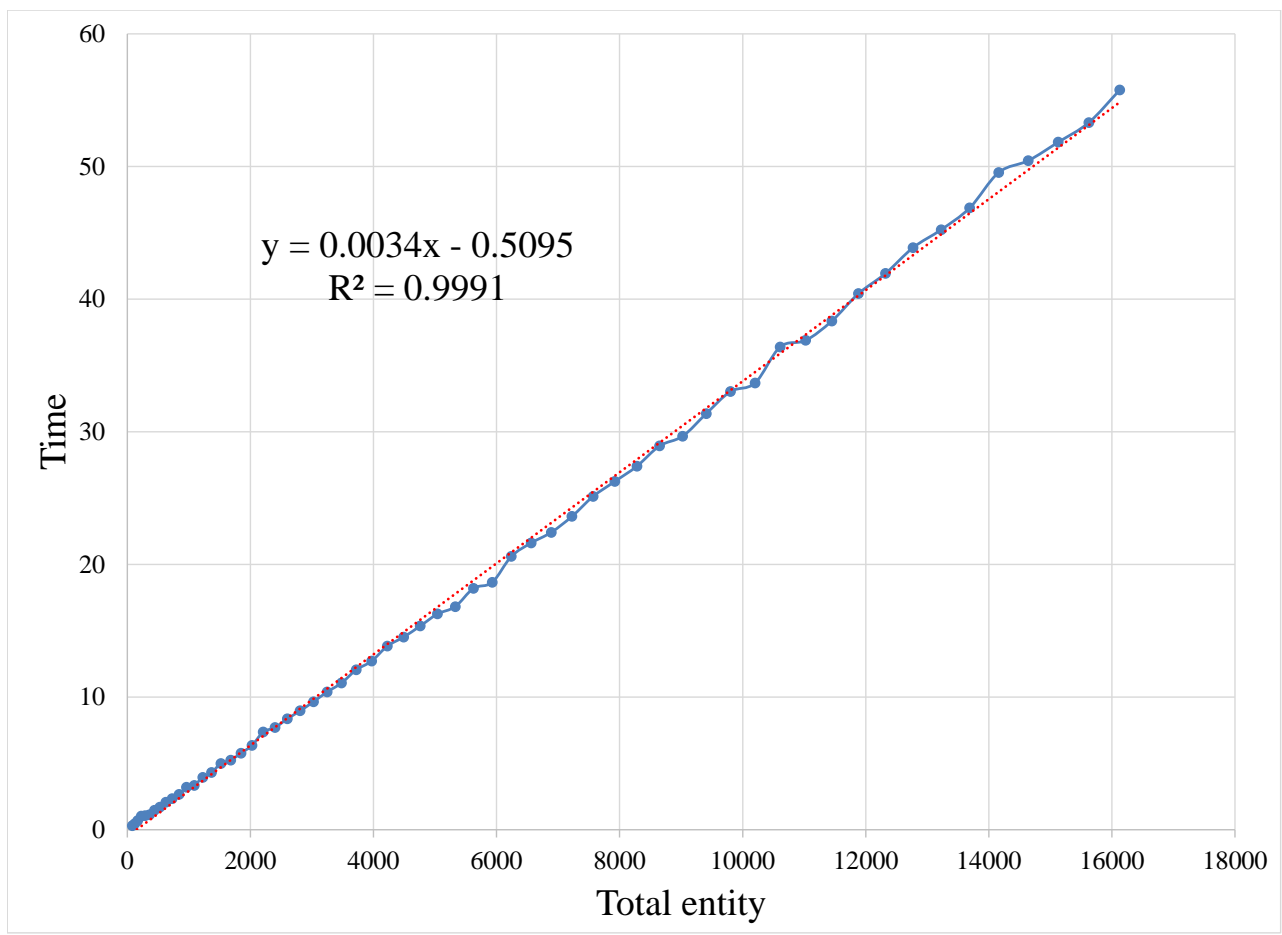

Figure 6: The time complexity for a function approximating (5) with a domain partitioned into a grid.

\subsection{Performance Comparison}

Consider the following additively separable PLQ function

$$
f\left(x_{1}, x_{2}\right)=x_{1}^{4}+x_{2}^{4} .
$$

We set $f_{1}\left(x_{1}\right)=x_{1}^{4}$ and $f_{2}\left(x_{2}\right)=x_{2}^{4}$. We use the plq_build function from the CCA package [Luc13a] to compute a quadratic approximation of $f_{1}$ with an univariate PLQ function. Then we combine the approximation to obtain an approximation of the bivariate function $f$.

Next we compute all the entities and represents them using GPH matrices. We build the hypermatrix $H_{p}$ and compute the neighbor matrix $N_{M}$. Then we apply our algorithm and compute an approximation of

$$
f^{*}\left(s_{1}, s_{2}\right)=f_{1}^{*}\left(s_{1}\right)+f_{2}^{*}\left(s_{2}\right),
$$

where $f_{1}^{*}\left(s_{1}\right)=\left(\frac{3}{4}\right)^{\frac{4}{3}} s_{1}^{\frac{4}{3}}$ and $f_{2}^{*}=f_{1}^{*}$. We increase the number of pieces by increasing the size of the grid and measure the time for computing the conjugate of $f$. Figure 6 illustrates the linear time complexity of our algorithm.

We compare the performance of our proposed algorithm with the algorithm developed in [Jak13] on Figure 7. For the algorithm developed in [Jak13], a linear least-square regression results in $R^{2}=$ 0.91 vs. $R^{2}=0.99$ for a quadratic regression i.e. the implementation tested on the specific example 


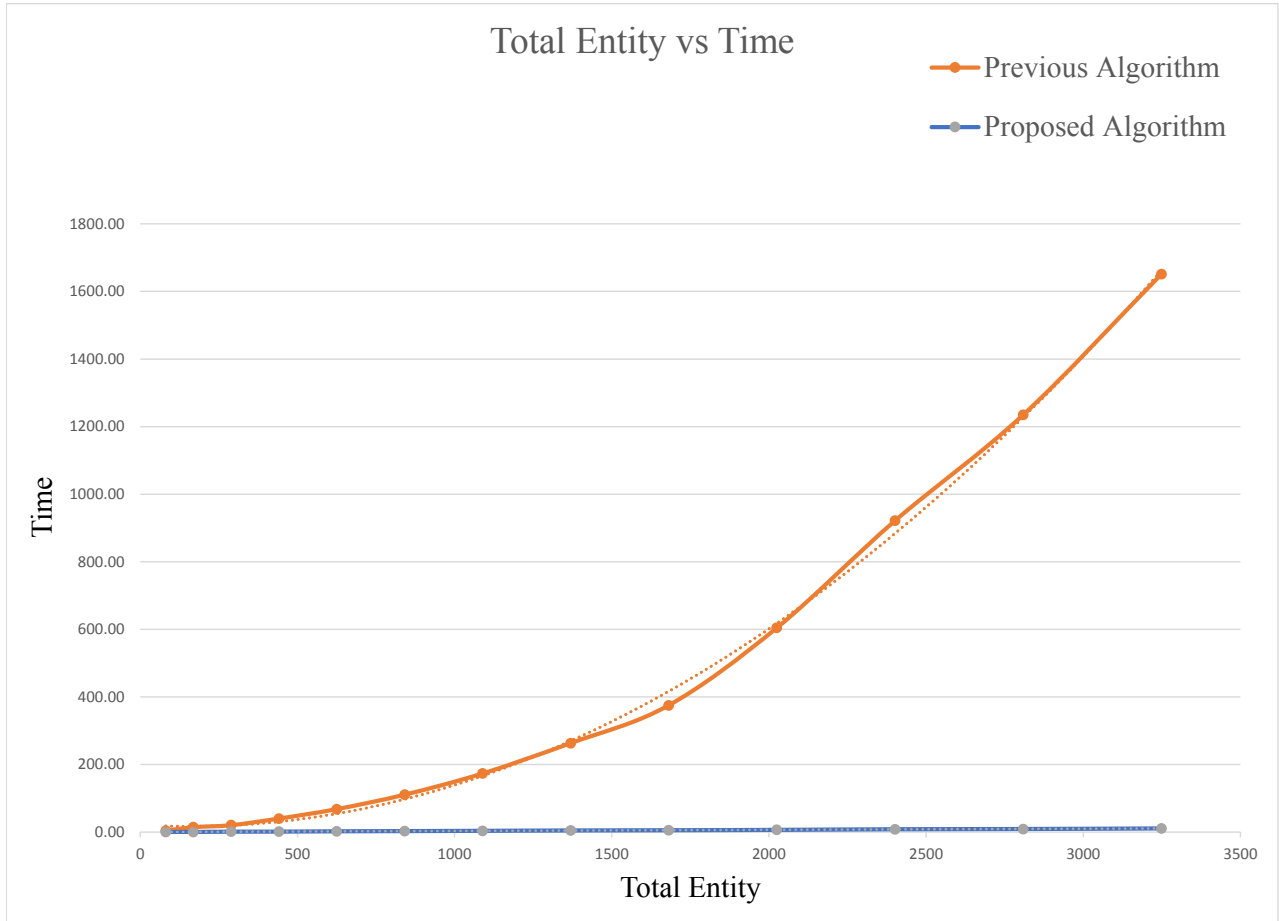

Figure 7: The time complexity for computing the conjugate of the a PLQ function approximating (5) using the algorithm from [Jak13] and the proposed algorithm .

runs in quadratic time. (We use the standard notation $R^{2}$ for the coefficient of determination that measures how close the data is to the regression line.) By contrast, a linear least-square regression fitted to the computation time of our algorithm gives $R^{2}=0.99$ thereby validating our linear computation time.

We run all numerical experiments on a Core(TM) i5 processor, 64 bit OS, 8.00 GB RAM, 2.40 GHz HP Pavilion x360 laptop, running Windows 10. The implementation of the algorithm is done using Scilab version 5.5.2. We performed the numerical experiment several times and obtained similar results each time.

The implementation of the algorithm from [Jak13] that we tested was a pure Scilab code that did not include the improvement of using the half-edge data structure provided in an external library. At the price of considerable complexity and a loss in portability, the [Jak13] algorithm can be implemented in log-linear time. However, our new algorithm would still be faster (linear-time) and much simpler.

\section{Conclusion and future work}

We proposed the first linear-time algorithm to compute the conjugate of a proper convex lsc bivariate PLQ function. That performance was achieved by taking advantage of adjacency information. 
Further simplification was made by using graph-matrix calculus. An example provides evidence of the significant speedup of our algorithm compared to previous work.

Future work includes developing graph-matrix calculus based algorithms for computing other convex transforms like the proximal average, the Moreau envelope, the addition and the scalar multiplication for bivariate PLQ functions. The implementation for functions defined on $\mathbb{R}^{d}$ would also be of interest.

\section{Acknowledgements}

This work was supported in part by Discovery Grants \#298145-2013 (Lucet) from NSERC, and The University of British Columbia, Okanagan campus. Part of the research was performed in the Computer-Aided Convex Analysis (CA2) laboratory funded by a Leaders Opportunity Fund (LOF, John R. Evans Leaders Fund - Funding for research infrastructure) from the Canada Foundation for Innovation (CFI) and by a British Columbia Knowledge Development Fund (BCKDF).

\section{References}

[AMO93] Ravindra K. Ahuja, Thomas L. Magnanti, and James B. Orlin. Network flows. Prentice Hall Inc., Englewood Cliffs, NJ, 1993. Theory, algorithms, and applications.

[CGA] CGAL, Computational Geometry Algorithms Library. http://www.cgal.org.

[Con94] Scilab Consortium. Scilab, 1994. http://www.scilab.org.

[Fan63] K. Fan. On the Krein-Milman theorem. Convexity, 7:211-220, 1963.

[GJL14] Bryan Gardiner, Khan Jakee, and Yves Lucet. Computing the partial conjugate of convex piecewise linear-quadratic bivariate functions. Comput. Optim. Appl., 58(1):249$272,2014$.

[GL10] Bryan Gardiner and Yves Lucet. Convex hull algorithms for piecewise linear-quadratic functions in computational convex analysis. Set-Valued Var. Anal., 18(3-4):467-482, 2010 .

[GL11] Bryan Gardiner and Yves Lucet. Graph-matrix calculus for computational convex analysis. In Heinz H. Bauschke, Regina S. Burachik, Patrick L. Combettes, Veit Elser, D. Russell Luke, and Henry Wolkowicz, editors, Fixed-Point Algorithms for Inverse Problems in Science and Engineering, volume 49 of Springer Optimization and Its Applications, pages 243-259. Springer New York, 2011.

[GL13] Bryan Gardiner and Yves Lucet. Computing the conjugate of convex piecewise linearquadratic bivariate functions. Math. Prog., 139(1-2):161-184, jun 2013.

[Goe08] Rafal Goebel. Self-dual smoothing of convex and saddle functions. J. Convex Anal., 15(1):179-190, 2008.

[Her16] Cristopher Hermosilla. Legendre transform and applications to finite and infinite optimization. Set-Valued Var. Anal., 24(4):685-705, 2016. 
[HUL93] Jean-Baptiste Hiriart-Urruty and Claude Lemaréchal. Convex Analysis and Minimization Algorithms II, volume 306 of Grundlehren der Mathematischen Wissenschaften [Fundamental Principles of Mathematical Sciences]. Springer-Verlag, Berlin, 1993. Vol II: Advanced theory and bundle methods.

[Jak13] Khan Md. Kamall Jakee. Computational convex analysis using parametric quadratic programming. Master's thesis, University of British Columbia, 2013.

[Knu73] D. E. Knuth. The Art of Computer Programming, Volume 3: Sorting and Searching. Series in computer-science and information processing. Addison-Wesley, 1973.

[Luc06] Yves Lucet. Fast Moreau envelope computation I: Numerical algorithms. Numer. Algorithms, 43(3):235-249, November 2006.

[Luc10] Yves Lucet. What shape is your conjugate? A survey of computational convex analysis and its applications. SIAM Rev., 52(3):505-542, 2010.

[Luc13a] Yves Lucet. Computational Convex Analysis library, 1996-2013.

[Luc13b] Yves Lucet. Techniques and open questions in computational convex analysis. In Computational and analytical mathematics, volume 50 of Springer Proc. Math. Stat., pages 485-500. Springer, New York, 2013.

[PS11] Panagiotis Patrinos and Haralambos Sarimveis. Convex parametric piecewise quadratic optimization: Theory and algorithms. Automatica, 47(8):1770 - 1777, 2011.

[PW16] C. Planiden and X. Wang. Strongly convex functions, moreau envelopes, and the generic nature of convex functions with strong minimizers. SIAM J. Optimiz., 26(2):1341-1364, 2016.

[Roc70] R. T. Rockafellar. Convex Analysis. Princeton University Press, 1970.

[RW98] R. T. Rockafellar and R. J.-B. Wets. Variational Analysis. Springer-Verlag, Berlin, 1998. 